\title{
Telemedicine in the era of COVID-19
}

(BIRDEM Med J 2020; 10, COVID Supplement: 1-3)

Telemedicine: World Health Organization (WHO) has defined telemedicine as "the delivery of healthcare services, where distance is a critical factor, by all healthcare professionals using information and communication technologies for the exchange of valid information for diagnosis, treatment and prevention of disease and injuries, research and evaluation and for the continuing education of healthcare providers".

Tele health: Telemedicine is sometimes used as a synonym to telehealth. Although WHO uses telemedicine to describe all aspects of health care including preventive care, many organizations use the term 'telemedicine' only to describe remote clinical services and 'telehealth' to include non-clinical applications like administration and providing education and health information for the common people.

Background: Modern information and communication technologies such as computers, internet and cell phones, seek and exchange information, have great potential to help address contemporary global health problems. Telemedicine, in its modern form, started in the 1960s.

In Bangladesh, telemedicine can play a particularly important role in cases where there is no need for the patient to physically see the doctor for regular routine check-ups or continuous monitoring. It can also reduce the burden on the secondary and tertiary hospitals. Disasters and pandemics pose unique challenges in providing health care. A telemedicine visit can be conducted without exposing staff to virus/infection in times of such outbreaks. In Bangladesh, till now there is no legislation or guidelines on the practice of telemedicine. The professional judgment of a Registered Medical Doctor should be the guiding principle for all telemedicine consultation.

Tools for telemedicine: Registered medical doctors may use any telemedicine tool suitable for carrying out technology-based patient consultation e.g. telephone, video, devices connected over LAN, WAN, internet, mobile or landline phones. Chat platforms like
WhatsApp, Facebook Messenger, or mobile apps or internet based digital platforms for telemedicine or data transmission systems like e-mail/Skype/fax etc. Irrespective of the tool of communication used, the core principles remain the same.

Delivery of telemedicine can come with three distinct domains: Live video (synchronous), store and forward (asynchronous) and remote patient monitoring. According to the Mode of Communications it can be Video (Telemedicine facility, Apps, Video on chat platforms, Skype/Facetime etc.), Audio (phone, VOIP, Apps etc.), Text based: 1. Telemedicine chat based applications (specialized telemedicine smartphone Apps, Websites, other internet-based systems etc.) 2. General messaging/text/chat platforms (WhatsApp, Google Hangouts, Facebook Messenger etc.) 3. E-mail, Fax etc.

Telemedicine services should however be avoided for emergency care when alternative in-person care is available and telemedicine consultation should be limited to first aid, life-saving measure, counselling and advice on referral.

Seven elements to be considered in any telemedicine consultation: 1. Context: Identification of registered medical doctor and identification of patient 3 . Mode of Communication 4. Consent (The consent must be explicit ) 5. Type of consultation (First and follow up consultation) 6. Patient evaluation 7. Patient management (health education, counseling \& medication).

Prescription should be with a diagnosis/provisional diagnosis. Prescription can be sent via any media with sign or e-sign. Prohibited medicine should not be prescribed. Electronic systems used will incorporate network and software security protocols to protect the confidentiality of patient identification and imaging data and will include measures to safeguard the data and to ensure its integrity against intentional or unintentional corruption. 
Expected benefits: Improved access to medical care by enabling a patient to remain in his/her place at a remote site, thereby improving access, equity and efficiency in the health system. Telemedicine can be beneficial to 1. patients in isolated communities and hard to reach locations, 2. vulnerable and old age group of people specially the bedridden patients whose mobilization is a difficult job, 3. remote patient monitoring through mobile technology can reduce the need for outpatient visits and enable remote prescription verification and drug administration, significantly reducing the overall cost of medical care, 4 . telemedicine also allows family physicians/general practitioners to consult with their peers and clinical experts when needed.

Possible risks: As with any medical procedure, there are potential risks associated with the use of telemedicine. These risks include, but may not be limited to: in rare cases, information transmitted may not be sufficient (e.g. poor resolution of images) to allow for appropriate medical decision making by the physician and consultant(s), delays in medical evaluation and treatment could occur due to deficiencies or failures of the equipment, in rare instances, security protocols could fail, causing a breach of privacy of personal medical information, in very rare cases, a lack of access to complete medical records may result in adverse drug interactions or allergic reactions or other judgment errors.

In this era of widespread digitalization and electricity, we still have some problem of utilization of these services which hampers telemedicine. The present outbreak of a coronavirus-associated acute respiratory disease called coronavirus disease 19 (COVID-19) is the third documented spillover of an animal coronavirus to humans that is causing a major epidemic in the last 2 decades. Now that COVID-19 is affecting us and our patients directly. COVID-19 is proving to be more, leading to 10 times as many cases in one-quarter of the time. Aim now is to slow its spread to avoid overwhelming the ability of our health care system to handle sick patients. Without proper containment measures, the fear is that hospitals will be overrun with COVID-19 cases. Not only does this limit our ability to treat seriously ill patients infected with the virus but it also could prevent uninfected individuals suffering from more mundane life-threatening conditions, such as myocardial infarction and stroke, from receiving timely treatment that they would routinely get in "normal" times.

Telemedicine can help: Telemedicine has the potential to help by permitting mildly ill patients to get the supportive care they need while minimizing their exposure to other acutely ill patients. To encourage the telemedicine approach, nearly all health plans and large employers offer some form of coverage for telemedicine services. The use of telemedicine has increased over the last 2 to 3 years. In response to the current COVID19 situation, the Centers for Medicare \& Medicaid Services and commercial health plans largely have increased utilization of telemedicine.

Office-based encounters to isolate providers: The use of telemedicine can allow providers who are older and who may have an underlying health condition to avoid contact with potentially infected patients. The provider would need a computer, tablet or smart phone for 2-way video interaction with patients and the office nurse could be trained to be a tele facilitator. For established patients where a physical examination is not required, media of communication platform would work. In such situations, if a procedure is needed, patients could even be seen from their home if they have the appropriate video equipment. If non-high-risk providers are present in the office, low-risk procedures such as skin testing can be performed.

For routine telemedicine video visits, many of the requirements have been waived during this time. Therefore, physicians can treat either new or established patients via telehealth video or telephone and are billable.

\section{Home-based video encounters for triage:}

Telemedicine also can be used to assess and triage for COVID-19. This type of encounter should be videobased and must be initiated by the patient to be billable. Although a facilitated visit may permit a physical examination to be performed, it also increases the risk of exposure to COVID-19 for patients and health care workers. With a home-based video interaction, the patient can have an interaction with a provider, who, in addition to obtaining a thorough history of symptoms and exposure risk, can perform an observational assessment. This assessment should include the following: temperature with a home thermometer, 
observation of general appearance, noting if the patient is ill appearing, is exhibiting diaphoresis, pallor, or flushing, calculation of respiratory rate, observation of respirations and deep breath and whether there is use of accessory respiratory muscles, labored breathing, interrupted speech, presence or absence of cough; dry or productive, observation of the oropharynx, with assessment of oropharyngeal erythema, exudate, enlarged or absent tonsils or lesions, patient-directed palpation of anterior and posterior cervical chains to assess for presence or absence of prominent lymphadenopathy.

Clinicians should use their judgment as to whether the patient is appropriate for COVID-19 testing. Priority should be given to patients with chronic medical conditions, individuals older than 65 years and those who have come into contact with a COVID-19 positive patient within 14 days. The patient can be directed to the appropriate facility for testing, home testing can be arranged, or if the patient is acutely ill, an emergency protocol should be in place with transfer to the nearest emergency department. Appropriate state and local reporting authorities should be contacted, just as if they had been seen in the office setting.

Telemedicine for management of chronic conditions: Telemedicine can be used for ongoing management of chronic diseases such as asthma and immunodeficiency, particularly during a time when social distancing is encouraged. Individuals with these conditions are particularly susceptible to COVID-19 and medication compliance and disease optimization are important ways to mitigate severity. Telemedicine can serve as a safe and effective alternative to in-person care. Recent studies have demonstrated similar health outcomes for patients whether delivered in person or synchronously by a remote provider for various conditions including asthma. A 2015 Cochrane systematic review examined the impact of telehealth involving remote monitoring or videoconferencing compared with in-person or telephone visits for chronic conditions including diabetes and congestive heart failure. This review found similar health outcomes for patients with these conditions. ${ }^{1}$

Recent surveys demonstrated that patients are willing to use telehealth but barriers still exist, namely: (1) at a time of need, many people revert to what they are used to doing and the way in which they previously interacted with the health care system. (2) patients would prefer that they see their own provider through telemedicine versus someone with whom they do not have a previously established relationship. (3) patients may be unaware that they have telemedicine as an option and do not know how to access it, this appear to be major obstacle in our practice.

Health plans, employers, hospital systems, and media outlets should work to overcome these barriers by (1) educating people that telemedicine is an effective alternative and safer under the current circumstances, (2) expanding network reimbursement coverage for physicians to see their patients through telemedicine, (3) making people aware that a telemedicine benefit exists, with step-by-step instructions on how it can be accessed, (4) helping people understand how telemedicine works and (5) continuing to reduce cost barriers to accessing telemedicine.

To promote the use of telemedicine in the age of COVID-19, various online resources have been developed both from regulatory agencies and commercial enterprenour.

So, although the presence of a pandemic is an unfortunate, though inevitable occurrence, it is also an opportunity to set up an infrastructure for providing care using telemedicine. Once the current pandemic is over, telemedicine can continue to be used to provide more convenient, cost-effective care to patients. In this way, we will already be prepared for the next, inevitable, infectious disease to emerge.

\section{REFERENCE}

1. Flodgren G, Rachas A, Farmer AJ, Inzitari M, Shepperd S. Interactive telemedicine: effects on professional practice and health care outcomes. Cochrane Database Syst Rev 2015; CD002098. [PMC free article] [PubMed] [Google Scholar 